\title{
Forstwirtschaft im schweizerischen Mittelland - wirtschaftsgeographische Analyse als wirtschaftspolitische Entscheidungsgrundlage
}

\author{
Martin Boesch
}

Im vorliegenden Artikel wird eine Untersuchung zusammengefaßt, die vor einiger Zeit im Auftrage eines forstwirtschaftlichen Ingenieurbüros durchgeführt wurde. Diese Studie scheint zu zeigen, daß praxisorientierte wirtschaftsgeographische Arbeiten durchaus relevante Grundlagen für wirtschaftspolitische Entscheide abgeben können - eine Tatsache, die nicht nur in akademischen Kreisen bisweilen übersehen wird.

\section{Auftrag}

In der Originalstudie ging es darum, die Realisierbarkeit einer neuen Forstbewirtschaftungsform an Hand eines konkreten Beispiels zu untersuchen und daraus ein allgemein verwendbares Beurteilungsschema abzuleiten.

Dieser Auftrag ist vor dem folgenden Hintergrund zu sehen: Die Forstwirtschaft in der Schweiz ist, beurteilt an der aktuellen gesamtwirtschaftlichen Situation, durch ausgesprochen ungünstige Voraussetzungen charakterisiert. Als entsprechende Stichworte müssen hier genügen: Verknappung der Arbeitskräfte, Verlagerung der Produktion bereichsmäßig aus den primären in sekundäre und tertiäre Zweige, sowie regional aus den ländlichen Entleerungsräumen in die städtischen Agglomerationsgebiete; Produktivitätssteigerungen durch Mechanisierung, Rationalisierung und überbetriebliche $\mathrm{Zu}$ sammenarbeit in vielen Wirtschaftszweigen sind wichtige Ursachen dieser Produktionsverlagerungen.

Da der Wald neben der Wirtschaftsfunktion im engeren Sinne auch eine öffentliche Aufgabe (Schutzund Erholungsfunktion) erfüllt, besteht aus ökologischen und landschaftshygienischen Gründen ein vitales allgemeines Interesse an einer Waldbewirtschaftung, deren Intensitätsgrad gegenüber heute eher wieder gesteigert werden sollte.

Im schweizerischen Mittelland wird nun seit einigen Jahren versucht, durch Waldumlegungen und großzügige Erschließungen die Produktionsgrundlagen zu verbessern. Die Betriebsform bleibt dagegen durch diese Maßnahmen weitgehend unberührt; wenig erstaunlich daher, daß verschiedentlich neue Konzepte der Forstbewirtschaftung diskutiert wurden. Eine solche Möglichkeit soll nun im folgenden skizziert und auf ihre Realisierungschancen beurteilt werden.

\section{Das Konzept der Forstbetriebs-Unternehmung}

Die Idee der Forstbetriebs-Unternehmung als einer potentiellen Alternative zur traditionellen Forstbewirtschaftung kann hier nur ganz knapp skizziert werden. $\mathrm{Da}$ in diesem Zusammenhang hauptsächlich die regionalen gesamtwirtschaftlichen Aspekte interessieren, soll hier insbesondere nicht darauf eingegangen werden, inwieweit die neue Konzeption betriebswirtschaftliche Vorteile bietet und anderseits forstpolitische und juristische Kontroversen verursacht.

Es geht im Prinzip darum, daß eine unternehmerisch geführte "Forstbetriebs-Gesellschaft» von einer ganzen Reihe von (öffentlichen und privaten) Waldbesitzern eines größeren Gebietes ${ }^{1}$ den langjährigen Auftrag zur selbstverantwortlichen integralen Forstbewirtschaftung erhält. Insbesondere besteht die Absicht, mit permanent angestellten Arbeitskräften die anfallenden waldbaulichen und Erntemaßnahmen ${ }^{2}$ über die bestehenden Besitzesgrenzen hinweg vorzunehmen, ohne daß freilich der alte Besitzstand in irgendeiner Weise umgelegt oder zusammengeworfen würde. Der Reingewinn beziehungsweise -verlust wird nach einem bestimmten Schlüssel zwischen Unternehmung (Unternehmergewinn) und Besitzer (Dividende) verteilt. Die einzelnen Waldbesitzer partizipieren gemäß ihren Anteilen (Bonitäts- und Bestandeswert der eingebrachten Parzellen) an diesem Erfolg der Betriebsführung. Damit wird deutlich, daß der Waldbesitz in diesem Konzept als reine Kapitalanlage betrachtet wird.

\section{Idee und Realisierbarkeit - eine Fallstudie}

Für die Untersuchung wurde nun ein Gebiet entsprechender Größe im Zürcher Weinland nach folgenden Gesichtspunkten eingehend analysiert:

- technische und betriebswirtschaftliche Verhältnisse der Forstwirtschaft; 
- gesamtwirtschaftliche Situation des Gebietes (insbesondere Stellung der Landwirtschaft) und Bedeutung der Forstwirtschaft in diesem Kontext;

- mutmaßliche Entwicklung der Region und $\mathrm{Zu}$ kunft der Forstbewirtschaftung in diesem Gesamtrahmen.

Zusammengefaßt ergab diese Analyse folgendes Bild:

1. Der waldbauliche Zustand als Resultat der effektiv vollzogenen Waldbewirtschaftung wird als mittelmäßig bis gut beurteilt.

2. Mehr als die Hälfte der Gesamtwaldfläche ist Privatwald, wovon Dreiviertel von den Bauern der Region bewirtschaftet werden, allerdings zuwenig intensiv vom rein forstlichen Gesichtspunkt aus. - Starke parzellare Zersplitterung und mangelhafte Erschließung mit Waldstraßen, die durch Lastwagen befahren werden können, sollen durch die Melioration behoben werden, die gegenwärtig durchgeführt wird.

3. Die kommunalen Forstorganisationen arbeiten intensiv und recht effizient.

4. Eine Sortimentsanalyse zeigt, daß der Brennholzanteil etwa bei einem Viertel der Nutzungsmenge liegt und fast ausschließlich der Selbstversorgung dient. Stamm- und Industrieholz gelangen auf den Markt.

5. Der Anteil der primären Erwerbstätigkeit im ganzen Gebiet ist außerordentlich hoch. Aus der Landwirtschaft resultiert knapp die Hälfte der gesamten regionalen Nettowertschöpfung. Die Betriebsgrößen (durchschnittlich $10 \mathrm{ha}$ ) sind bei leicht steigender Tendenz immer noch bescheiden. Neben den Vollerwerbsbetrieben behaupten sich eigenartigerweise die Nebenerwerbsbetriebe mit minimaler Kulturfläche. Entsprechend bleibt das bäuerliche Element in der Bevölkerung stark verankert, und es besteht ein ordentliches Reservoir an Arbeitskräften, die teilweise für die Waldarbeit verfügbar sind.

6. Obwohl nur ca. $4 \%$ der gesamten regionalen Nettowertschöpfung direkt aus der Waldarbeit stammen (ohne Holzverarbeitung in Sägereien usw.), ist für Dreiviertel aller Landwirtschaftsbetriebe die Privatwaldbewirtschaftung nicht nur bezüglich Arbeitsorganisation, sondern auch im Hinblick auf das Haushalteinkommen ein wesentlicher, integrierter Betriebsbestandteil.

7. Gestützt auf die Ergebnisse der zuständigen Regionalplanungsgruppe kann die mutmaßliche Entwicklung des Gebietes etwa folgendermaßen skizziert werden:

Zunehmende Betriebsgröße und sinkende Betriebszahl in der Landwirtschaft einerseits, steigende Bevölkerungszahl anderseits bedeuten eine Verlagerung der Beschäftigung aus dem primä- ren in sekundäre und tertiäre Sektoren. Da aber kaum genügend neue Arbeitsplätze in der Region selbst geschaffen werden, ist ein stark steigender Pendleranteil zu erwarten. Dies ist bei relativ weit entfernten Ballungs- und Arbeitszentren eine recht ungünstige Situation, so daß kaum mit einer stürmischen Entwicklung zu rechnen ist.

Im Blick auf die oben dargelegte unternehmerische forstwirtschaftliche Konzeption wird die Gesamtsituation des Gebietes wie folgt interpretiert:

- Weder aus ökologischen noch aus landschaftshygienischen Gründen drängt sich eine Intensivierung der Waldbewirtschaftung auf; dagegen wäre sie aus forstlichen Gründen erwünscht und durchaus vertretbar.

- Eine hypothetische Ertragsanalyse zeigt, daß die zukünftige Gewinnausschüttung an die Gemeinden die heutigen Betriebsüberschüsse aus den öffentlichen Waldungen kaum übertreffen dürfte.

- Den Privatwaldbesitzern würde eine Kapitalrente in der Höhe von höchstens $2 \%$ der Aktiven zufließen. Dies entspricht etwa 10 bis $20 \%$ des gegenwärtig erzielten Arbeitseinkommens der Bauern aus dem Wald.

- Im günstigen landwirtschaftlichen Gelände des Untersuchungsgebietes sind auch bei einer Verdoppelung der Betriebsgrößen zwei bis drei Arbeitskräfte je Betrieb kaum genügend ausgelastet, weitgehende Mechanisierung vorausgesetzt. So muß weiterhin mit zusätzlich verfügbarer bäuerlicher Arbeitszeit gerechnet werden.

- Bei der vorgeschlagenen unternehmerischen forstwirtschaftlichen Organisationsform fällt hingegen die bäuerliche Teilzeitarbeit im Walde weitgehend weg, so daß auf dieses Arbeitseinkommen verzichtet werden muß.

\section{Von der Fallstudie zu einem allgemeinen Bewer- tungsschema}

Ein konsistentes funktionales Gleichungssystem stellt zweifellos das Idealziel einer solchen Untersuchung dar. Aus verschiedenen Gründen war aber dieses Ziel für die vorliegende Studie unerreichbar. So wurde versucht, als erste Annäherung eine Reihe von relevanten Kriterien ${ }^{3}$ in einfachen Bewertungsfunktionen einzeln zu fassen und Kriterien mit ähnlicher Aussagerichtung zu Kriterienbündeln zusammenzufassen. Als Zielgröße (oder abhängige Variable) war immer die "Realisierungschance der Unternehmerkonzeption» zu wählen. Jedes der insgesamt fünf Kriterienbündel (mit je zwei bis vier Grundkriterien) stellt eine neue übergeordnete Variable dar, welche mit der Zielgröße prinzipiell posi- 


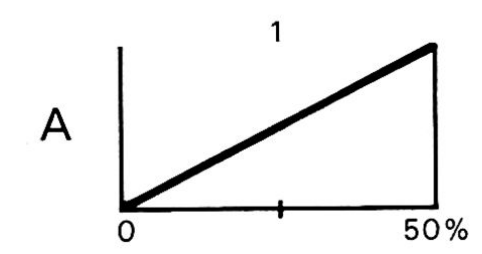

3
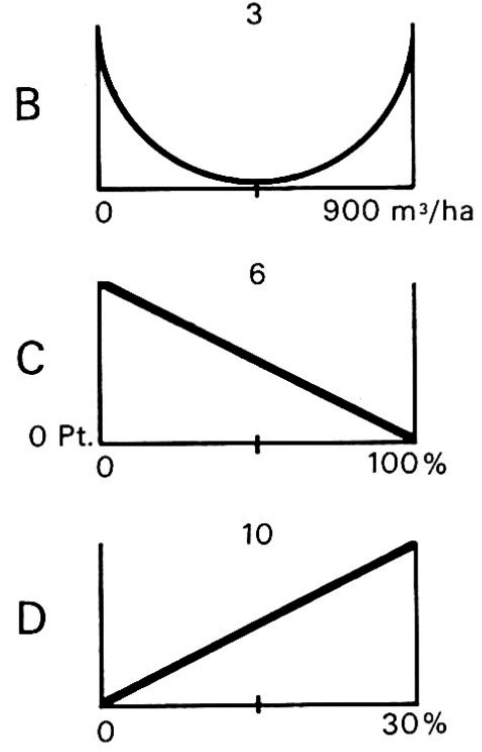

$E$

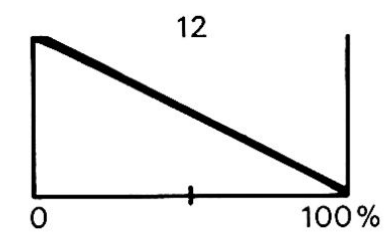

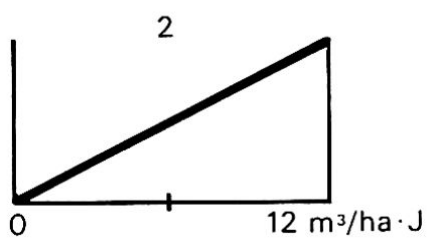
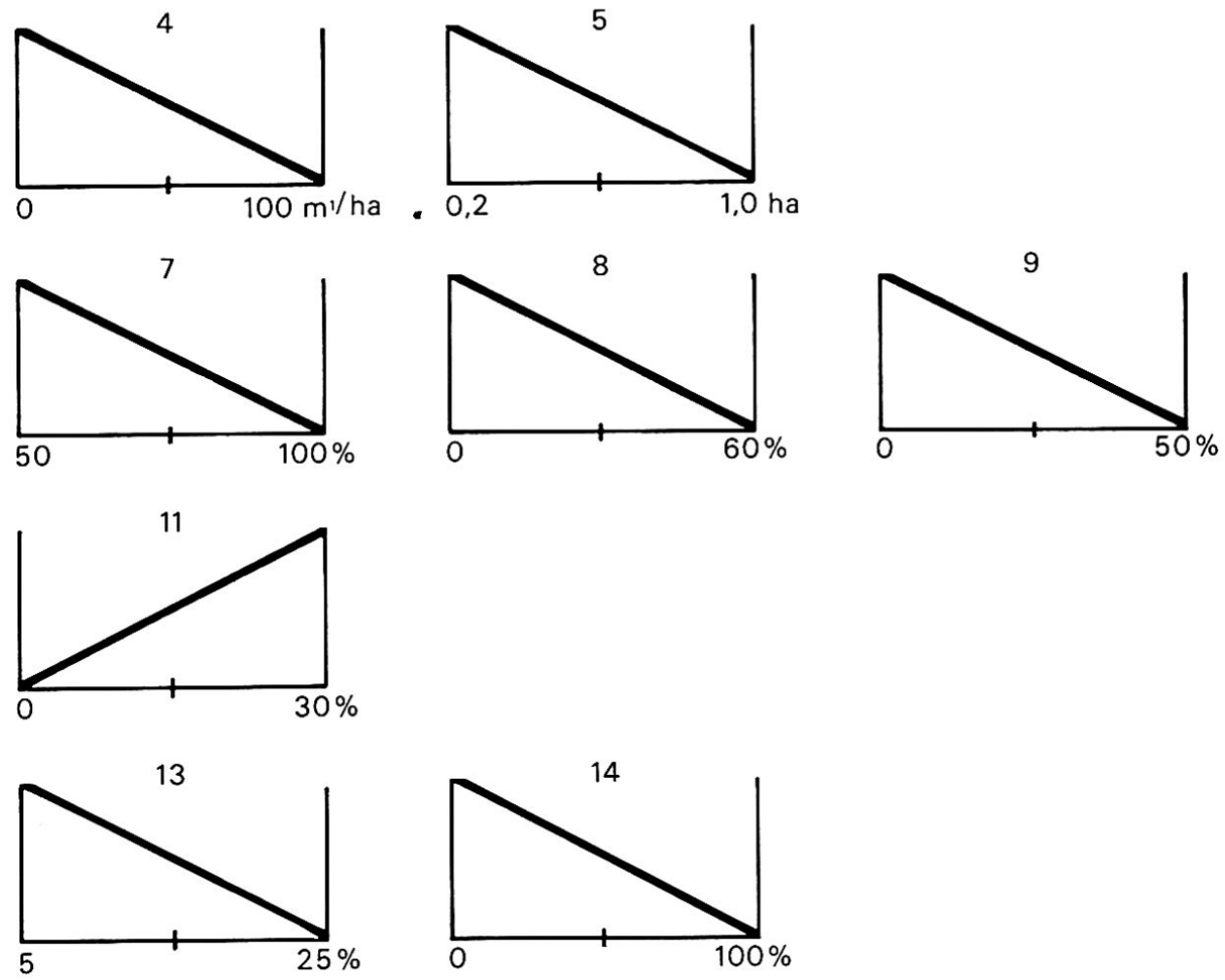

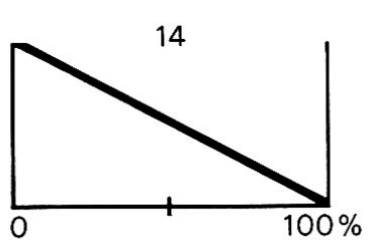

tiv oder negativ korreliert ist. Damit ergeben sich zwei verschiedene Interpretationen der Kriterien bzw. Kriterienbündel:

1. Bei positiver Korrelation handelt es sich um Variablen, welche die allgemeine Bedeutung des Waldes und der Forstwirtschaft im und für das Untersuchungsgebiet ausdrücken. Ist diese Bedeutung groß, so sind auch tendenziell das Interesse an einer wirkungsvollen Forstbewirtschaftung und damit die Realisierungschancen der neuen Konzeption groß. Kriterienbündel A und D.

2. Bei negativer Korrelation handelt es sich um Variablen, die verschiedene Aspekte der Intensität und Effizienz bisheriger und zukünftiger Waldbewirtschaftung ausdrücken. Ist diese Aktivität bereits groß, so ist das Interesse an und die Notwendigkeit zu Änderungen gering; damit sind die Realisierungschancen der neuen Konzeption klein. Kriterienbündel B, C und E.

Es ergibt sich, daß die einzelnen Kriterienbündel und ganz besonders die beiden Gruppen keineswegs gegeneinander "verrechnet" werden dürfen, denn ihre gegenseitige kausale Abhängigkeit und ihre quantitativen Beziehungen sind noch nicht geklärt. Mit andern Worten: Das Problem der Gewichtung der einzelnen Kriterienbündel ist noch offen. - Für die einzelnen Kriterienbündel dagegen wurden durchschnittliche Punktzahlen ermittelt, weil innerhalb der Bündel mit einer einfachen Kompensation gerechnet wird.

Die Grenzwerte der einzelnen Bewertungsfunktionen sind entsprechend den Verhältnissen im schweizerischen Mittelland gewählt worden und damit nicht allgemein gültig. - Zur Punktbewertung ist zu bemerken, daß für jedes Kriterium 0 bis 10 Punkte resultieren können, wobei kleine Werte «geringe Realisierungschancen" und hohe Werte "große Realisierungschancen" bedeuten. Es ist natürlich außerordentlich schwierig, die Kriterien jeweils als isoliertes Phänomen zu beurteilen, doch ist dieses partielle Vorgehen eine Notwendigkeit in diesem Anfangsstadium der Formalisierung.

Im Detail spielte sich der Arbeitsprozeß wie folgt ab: Nachdem entschieden war, daß ein Kriterium relevant sei4 für die Zielvariable, mußte ein Faktor gefunden werden, der zwei Bedingungen erfüllte: $\mathrm{Er}$ mußte einerseits ein möglichst genaues $M a ß$ für das Kriterium sein, anderseits aber rasch und einfach 
für irgendein Gebiet zu bestimmen sein, vorzugsweise auf Grund bestehender statistischer Erhebungen.

Dies sei an einem Beispiel erläutert. Die Standortsqualität ist insofern ein relevantes Kriterium, als ein standortmäßig erstklassiges Gebiet für eine unternehmerische Aktivität bedeutend attraktiver ist als ein für Wald eher ungeeignetes Gebiet. Nun stellte sich die Frage, welcher rasch zugängliche Faktor ein einigermaßen adäquates $\mathrm{Maß}$ für die Standortsqualität (die nicht als solche festgelegt ist) abgeben würde. Schließlich wurde die mittlere Zuwachsleistung (in $\mathrm{m}^{3 / \mathrm{ha}} \cdot \mathrm{J}$ ) gewählt. Sie ist den Wirtschaftsplänen der öffentlichen Waldungen rasch und relativ zuverlässig zu entnehmen und kann normalerweise als für das ganze Gebiet repräsentativ betrachtet werden. Freilich ist der Zuwachs nicht nur von den Standortsfaktoren beeinflußt, sondern zum Beispiel auch von der Stärkeklassenverteilung der Bestände. Es wurde nun entschieden, daß dieser Ein- fluß normalerweise ohne Korrektur vernachlässigt werden kann. - Schließlich galt es, den generellen Funktionsverlauf und die Grenzwerte entsprechend den im schweizerischen Mittelland vorherrschenden Verhältnissen festzulegen, im Beispiel des Zuwachses eine einfache lineare Beziehung mit positiver Steigung und den Grenzwerten 0 und $12 \mathrm{~m}^{3 / h a} \cdot \mathrm{J}$ für 0 bzw. 10 Punkte.

Es ist selbstverständlich, daß sich das Anfangsstadium dieser Studie auch darin äußert, daß die so bestimmten Faktoren die Kriterien nicht mit aller wünschbaren Genauigkeit ausdrücken. Dies vor allem deshalb, weil stets die Verfügbarkeit der Daten und die Einfachheit des ganzen Vorgehens beachtet werden mußten.

Es folgen nun eine tabellarische Zusammenstellung der 14 Kriterien und der zugehörigen Maßfaktoren sowie die entsprechenden graphisch dargestellten Bewertungsfunktionen.
Kriterium

\section{A. Bedeutung des Waldes}

1. allgemeine Bedeutung des Waldes für Wirtschaft, Landschaftsstruktur und Bevölkerung

2. Standortsqualität

\section{B. Zustand des Waldes}

3. Nachhaltigkeit

4. Erschließung

\section{Parzellierungsgrad}

\section{Bewirtschaftung}

6. Nutzungsintensität

7. Sortiment ${ }^{4}$

8. Effizienz des kommunalen Forstdienstes

9. regionale Erwerbsstruktur6

\section{Bedeutung des öffentlichen Waldes}

10. Interesse der Gemeinde(n) am Wald

11. wirtschaftliches Potential der öffentlichen Waldungen 7

\section{E. Bedeutung des Privatwaldes}

12. Bauernwaldanteil8

13. Bedeutung des Waldes für die Landwirtschaft

14. Nutzung des Privatwaldes
Faktor

Maßeinheit

Waldfläche/Gesamtfläche des Gebietes

$\%$

Zuwachsleistung1

$\mathrm{m}^{3 / \mathrm{ha}} \cdot \mathrm{J}$
Vorrat 2

$\mathrm{m}^{3 / h a}$

Dichte des Straßen- und Wegnetzes in den

Wäldern

durchschnittliche Parzellengröße ${ }^{3}$

$\mathrm{m} 1 / \mathrm{ha}$

ha

Nutzung/Zuwachs ${ }^{1}$

$\%$

Anteil des Stamm- und Nutzholzes an der

Gesamtnutzung

$\%$

Betriebsüberschuß/Gesamteinnahmen aus

öffentlichen Waldungen

landwirtschaftliche Erwerbsbevölkerung/

gesamte aktive Bevölkerung

\section{$\%$}

$\%$

öffentlicher Wald/Gesamtgebiet

$\%$

potentieller Betriebsüberschuß aus öffentlichen Waldungen/Totaleinnahmen der Gemeinde

\section{$\%$}

Bauernwald/gesamte Privatwaldfläche

Bauernwaldfläche/gesamte waldfreie Kulturfläche 9

Flächenanteil der Eigenbewirtschaftung im Privatwald 10
$\%$

$\%$

Bemerkungen und Erläuterungen siehe Seite 201 


\section{Bemerkungen und Erläuterungen}

1 Die Zuwachsleistung gibt einigermaßen die Umweltbedingungen (Lage, Relief, Böden, Klima) wieder, freilich unter der Voraussetzung einer normalen Stärkeklassenverteilung. Idealer wäre eine "potentielle, standörtlich bedingte Zuwachsleistung», die aber nicht rasch und eindeutig erfaßbar ist.

$2 \mathrm{Zu}$ hohe Vorräte lassen auf überalterte Bestände, zu kleine Vorräte auf übernutzte Bestände schlieBen. Besser wäre eine Beurteilung der Stärkeklassenverteilung, doch stehen diese Daten meist nicht zur Verfügung.

3 Die Parzellengrößenverteilung wäre viel aussagekräftiger, jedoch nicht einfach genug.

4 Das Sortiment läßt Rückschlüsse zu auf Holzverwendung und Wirtschaftseinstellung (Selbstversorgung - Marktorientierung), und damit auf einen Aspekt der Bewirtschaftungsintensität.

5 Hier sind die Forstabrechnungen sehr kritisch zu prüfen auf überwälzte Ausgabenposten und damit überbewertete Betriebsergebnisse.

6 Die Erwerbsstruktur soll in erster Linie einen Hinweis auf das Potential an Arbeitskräften für die Waldarbeit geben.

7 Dies ist ein $\mathrm{Ma} ß$ dafür, wie stark der öffentliche Haushalt auf den Nettoeinnahmen aus dem Wald basieren könnte, falls die Möglichkeiten optimal ausgenützt wären. Als entsprechender Faktor wird der Anteil der potentiellen Betriebsüberschüsse an den Gesamteinnahmen der Gemeinden berechnet. Die potentiellen Betriebsüberschüsse ergeben sich aus den effektiv erzielten Gewinnen und der Nutzungsintensität, so daß gilt: $E_{\text {pot }}=E_{\text {eff }} \cdot Z / N$.

Der Einfluß der Effizienz ist damit nicht berücksichtigt, doch muß vorderhand diese erste einfache Annäherung genügen.

8 Dieses Kriterium wurde aus der Erfahrung gewählt, daß für die Realisierungsbeurteilung der Bauernwald vom restlichen Privatwald zu unterscheiden ist: Die Neigung, die Waldbewirtschaftung integral einer Unternehmung zu übertragen, ist im Bauernwald sehr gering, bei den übrigen Privatwaldbesitzern dagegen meist latent vorhanden.

9 Besser wäre die Beurteilung der Wertschöpfungsverhältnisse, doch sind diese Größen nur ganz grob schätzbar. Hier muß das rasch erfaßbare Flächenverhältnis genügen.

10 Die flächenmäßige Nutzungsintensität drückt besser aus als die mengenmäßige, wieviel Wald wirklich noch im Landwirtschaftsbetrieb integriert ist. $\mathrm{Da}$ viele Bauern nichtbäuerlichen Privatwald im Auftrag bewirtschaften, wurde hier auf die gesamte Privatwaldfläche Bezug genommen.

\section{Anwendung des Bewertungsschemas}

Am Beispiel desselben Gebietes, das in der Fallstudie analysiert wurde, soll nun noch die praktische Anwendung des Bewertungsschemas gezeigt werden. Diese Bewertung basiert vorwiegend auf allgemein zugänglichen Statistiken sowie den Wirtschaftsplänen der öffentlichen Waldungen. Daneben war aber auch eine spezielle Erhebung bei den Revierförstern nötig, um die Besitzes- und Bewirtschaftungsverhältnisse im Privatwald erfassen zu können. Ferner konnten gewisse Daten (ErschlieBung, Parzellierungsgrad) den Erhebungen für die Waldmelioration, die gegenwärtig durchgeführt wird, entnommen werden. Da diese Informationsquelle normalerweise nicht zur Verfügung steht, ist man für diese Faktoren auf Schätzungen angewiesen, die auf den Grundbuchplänen beruhen.

Die 14 Faktorwerte wurden im einzelnen wie folgt eingesetzt:

Faktor

A.

1. Waldanteil/Gesamtfläche (\%) 316

2. Zuwachs $\left(\mathrm{m}^{3 / \mathrm{ha}} \cdot \mathrm{J}\right) \quad 11.0 \quad 9$

7.5

B.

3. Vorrat ( $\left.\mathrm{m}^{3} / \mathrm{ha}\right)$

4250

4. Waldstraßendichte $\left(\mathrm{m}^{1 / \mathrm{ha}}\right)$

5. durchschnittliche Parzellengröße (ha)

$\begin{array}{lll}52 & 5 & 4.7\end{array}$

0.249

C.

6. Nutzung/Zuwachs (\%)

$66 \quad 3$

7. Stamm- und Industrieholz/ Nutzung (\%)

$75 \quad 5$

8. Betriebsüberschüsse/Einnahmen im öffentlichen Wald (\%)

$43 \quad 3$

9. landwirtschaftlich Beschäftigte/ Aktivbevölkerung (\%)

$47 \quad 1$

D.

10. öffentlicher Wald/Gesamtfläche $(\%)$

11. potentieller Betriebsüberschuß/

Totaleinnahmen der Gemeinde (\%) 6

E.

12. Bauernwald/Privatwald (\%) $74 \quad 3$

13. Bauernwaldfläche/gesamte waldfreie Kulturfläche (\%)

$\begin{array}{lll}18 & 4 & 3.3\end{array}$

14. Flächenanteil der Eigenbewirtschaftung im Privatwald (\%) $72 \quad 3$ 
Verbal kann diese Bewertung etwa wie folgt interpretiert werden:

A. Dem Wald kommt im Untersuchungsgebiet eine große quantitative und qualitative Bedeutung zu. Es besteht somit ein starker Anreiz für unternehmerische Tätigkeit sowie auch ein allgemeines öffentliches Interesse an gesamtwirtschaftlich optimaler Nutzung.

B. + C. Der Zustand der Wälder an sich ist gut, dagegen ist die Erschließung mittelmäßig und der Parzellierungsgrad sehr hoch. Dies soll durch die Melioration bedeutend verbessert werden. Die bisherige Bewirtschaftung ist recht intensiv, marktorientiert und effizient. Es sind genügend Arbeitskräfte vorhanden. Gesamthaft beurteilt ist die Notwendigkeit einer neuen Bewirtschaftungskonzeption nicht dringend.

D. Dem öffentlichen Wald kommt im Gesamtrahmen des Gemeindehaushaltes keine überragende Stellung zu. Das Interesse an einer Strukturänderung ist daher eher gering.

E. Der Privatwald ist stark in die Landwirtschaft integriert; er wird regelmäßig genutzt. Darin besteht ein gewisses Hindernis für die Realisierung der neuen Konzeption.

Gesamthaft betrachtet kann gesagt werden, daß die Realisierungschancen der Unternehmerkonzeption eher gering sind. Haupthindernisse dürften trotz der Bedeutung des Waldes die gute bisherige Bewirtschaftung durch die kommunalen Forstdienste sowie die starke Bedeutung des Bauernwaldes sein.

\section{Schlußbemerkungen}

Es hat sich im Laufe der Untersuchung gezeigt, daß für eine solche umfassende Neukonzeption der Waldbewirtschaftung nicht allein die forstwirtschaftlichen Verhältnisse maßgebende Kriterien sind. Die Forstwirtschaft ist derart in die gesamtwirtschaftliche Struktur integriert, daß weitreichende Entscheidungen nicht allein auf Grund der forstlichen Bedürfnisse und Anschauungen getroffen werden können. Vielmehr ist das Interesse der Öffentlichkeit sowie anderer betroffener Wirtschaftssektoren mitabzuwägen ${ }^{5}$. Dabei dürfen selbstverständlich bestimmte forstökologische und forstwirtschaftliche Normen nicht verletzt werden. Diese allgemeinen Bemerkungen treffen natürlich für alle wirtschaftspolitischen Entscheidungen zu: partikulären Interessen darf nur im Rahmen der Gesamtstruktur stattgegeben werden, was freilich nur bei konsistenter allgemeiner Wirtschaftspolitik möglich ist. Wirtschaftsgeographische Untersusuchungen dürften in dieser Situation bisweilen wertvoll sein, wenn es darum geht, sozio-ökonomische Strukturen in gegenseitiger Abhängigkeit und gleichzeitig in ihren Beziehungen zur Umwelt zu analysieren.

\section{Literatur}

Boesch M. und Szilagyi T.: Die Forstwirtschaft in der Region Stammheim/ZH. Unpublizierte Studie für Forest Consult, Zürich, 1970.

Fischer G.: Berechnungen und Vorausschätzungen regionaler Volkseinkommenszahlen in der Schweiz, 1950-1980. Struktur- und Regionalwirtschaftsstudien, Bd. 3, St. Gallen, 1969.

(Grob H. und) Boesch M.: Zur Lage der forstwirtschaftlichen Unternehmungen in der Schweiz. Allg. Forstzeitschrift, Nr. 28, München, 1970.

Guth, Lechner und Philipp: Zwischenbericht zur Regionalplanung Zürcher Weinland. Zürich, 1964. Huber A.: Der Privatwald in der Schweiz. Diss. ETH, Zürich, 1948.

Schiller G.: Beitrag zur generellen Planung der Forstwirtschaft. Diplomarbeit ETH, Zürich, 1968. Zelther J. et al.: Grundlagenplan für die Waldungen der Region Gäu. Eidg. Anstalt für das forstl. Versuchswesen, Birmensdorf, 1965.

Gemeindespiegel für Oberstammheim, Unterstammheim und Waltalingen, ORL-ETH, Zürich.

Statistisches Handbuch des Kantons Zürich, Heft 53 der Stat. Mitteilungen des Kt. Zürich, 1964.

Statistische Schriften des Schweiz. Bauernsekretariates: Ergebnisse von Buchhaltungserhebungen in Landwirtschaftsbetrieben, Nr. 76, 96 und 101, Brugg, 1962-1969.

Wirtschaftspläne der Gemeinden Oberstammheim, Unterstammheim und Waltalingen/Guntalingen. Oberforstamt, kantonale Volkswirtschaftsdirektion,

\section{Anmerkungen}

1 Als Grenzwert wird etwa eine minimale Waldfläche von 1000 ha angenommen.

2 Die Nutzungspolitik liegt ganz in den Händen der Unternehmung, im Interesse einer optimalen Sortimentserzeugung. Die Nachhaltigkeit soll sehr großräumig und langfristig gewährleistet sein.

${ }^{3}$ Diese Auswahl bedeutungsvoller Kriterien stützt sich weitgehend auf die Fallstudie; ihre Relevanz ist nur relativ gesichert. Insgesamt wurden schließlich 14 Kriterien in die Studie aufgenommen.

4 Weitgehend auf Grund der Felderfahrung, aus Diskussionen und manchmal gestützt auf Literaturhinweise.

5 In diesem Rahmen muß auch die Bedeutung der Waldmeliorationen beurteilt werden. Sie fördern eine Struktur der Waldbewirtschaftung, die vom forstlichen Standpunkte aus nicht optimal ist, sich dagegen gesamtwirtschaftlich durchaus rechtfertigt. 